\title{
Le genre Oxyanthus DC. (Rubiaceae) en Afrique de l'Ouest: description d'une nouvelle espèce
}

\author{
Olivier Lachenaud \& Bonaventure Sonké
}

\begin{abstract}
LACHENAUD, O. \& B. SONKÉ (2015). The genus Oxyanthus DC. (Rubiaceae) in West Africa: description of a new species. Candollea $70: 241-247$. In French, English and French abstracts. DOI: http://dx.doi.org/10.15553/c2015v702a10

A new species, Oxyanthus andjigae Sonké \& O. Lachenaud (Rubiaceae), from Ivory Coast and Sierra Leone, is described and illustrated. It is closely related to Oxyanthus racemosus (Schumach. \& Thonn.) Keay and Oxyanthus setosus Keay, and was previously confused with the latter species; however, it differs from both in the longer corolla tube $(18-19.5 \mathrm{~cm}$ vs (7-)11-15.5 cm) and the longer hairs on the twigs (1.5-2 mm long vs. $\leq 1 \mathrm{~mm}$ in Oxyanthus setosus and $\leq 0.5 \mathrm{~mm}$ in Oxyanthus racemosus). From Oxyanthus setosus it is also separated by the distribution, the latter species occuring from Nigeria to Gabon. The species is assessed as "Endangered" following IUCN Categories and Criteria. A new identification key to West African species of Oxyanthus DC. is presented; Oxyanthus oliganthus K. Schum. is newly reported from Côte d'Ivoire and Ghana.
\end{abstract}

\section{Résumé}

LACHENAUD, O. \& B. SONKÉ (2015). Le genre Oxyanthus DC. (Rubiaceae) en Afrique de l'Ouest: description d'une nouvelle espèce. Candollea 70: 241-247. En français, résumés anglais et français. DOI: http://dx.doi.org/10.15553/c2015v702a10

Une nouvelle espèce, Oxyanthus andjigae Sonké \& O. Lachenaud (Rubiaceae), de Côte d'Ivoire et de Sierra Leone, est décrite et illustrée. Elle est voisine d'Oxyanthus racemosus (Schumach. \& Thonn.) Keay et d'Oxyanthus setosus Keay, et avait été jusqu'à présent confondue avec ce dernier; cependant, elle diffère de ces deux espèces par le tube de la corolle plus long (18-19,5 cm au lieu de (7-)11-15,5 cm) et les rameaux à poils plus longs $(1,5-2 \mathrm{~mm}$ contre $\leq 1 \mathrm{~mm}$ chez Oxyanthus setosus et $\leq 0.5 \mathrm{~mm}$ chez Oxyanthus racemosus). Elle diffère également d'Oxyanthus setosus par sa distribution, ce dernier étant connu du Nigeria au Gabon. L'espèce est évaluée comme «En Danger» selon les Catégories et Critères de l'UICN. Une nouvelle clé du genre Oxyanthus DC. pour l'Afrique de l'Ouest est proposée. Oxyanthus oliganthus K. Schum. est signalé pour la première fois de Côte d'Ivoire et du Ghana.

\section{Keywords}

RUBLACEAE - Oxyanthus - West Africa - Côte d'Ivoire - Sierra Leone - Taxonomy - New species

\footnotetext{
Adresses des auteurs:

OL: Jardin Botanique Meise, Domein van Bouchout, 1860 Meise, Belgique et Herbarium et Bibliothèque de Botanique africaine, C.P. 169, Université Libre de Bruxelles, av. F. Roosevelt 50, B-1050, Bruxelles, Belgique. E-mail: olivier.lachenaud@jardinbotaniquemeise.be

BS: Plant Systematic and Ecology Laboratory, Higher Teacher's Training College, University of Yaoundé I, Yaoundé, Cameroon et Missouri Botanical Garden, Africa \& Madagascar Department, P.O. Box 299, St. Louis, Missouri 63166-0299, U.S.A. et Herbarium et Bibliothèque de Botanique africaine, C.P. 169, Université Libre de Bruxelles, Av. F. Roosevelt 50, B-1050, Bruxelles, Belgique.

Soumis le 26 juin 2015. Accepté le 12 Septembre 2015.

Edité par M. W. Callmander \& P. Bungener
} 


\section{Introduction}

Le genre Oxyanthus DC. (Rubiaceae) compte environ 35 espèces, toutes originaires d'Afrique tropicale (SonkÉ, 1999). Ce sont des arbustes ou de petits arbres croissant en sous-bois dans les régions forestières humides, et plus rarement dans les galeries forestières en savane.

Le genre était traditionnellement rattaché à la tribu des Gardenieae, définie dans un sens très large (KeAy, 1958; Hallé, 1970). Toutefois, à la lumière de données moléculaires, Mouly et al. (2014) l'ont récemment transféré dans la nouvelle tribu des Sherbournieae; celle-ci regroupe quatre genres africains (Oxyanthus, Atractogyne Pierre, Mitriostigma Hochst. et Sherbournia G. Don) caractérisés par leurs graines à surface striée, alors que les Gardenieae s.s. ont les graines lisses. Le genre Oxyanthus se distingue aisément de ses trois proches parents par la corolle à tube allongé et très étroitement cylindrique, et les étamines exsertes (semi-exsertes chez O. schumannianus De Wild. \& T. Durand). Ses autres caractères notables sont les stipules persistantes, le calice réduit à dents courtes, et les inflorescences pluriflores et dressées.

Le genre Oxyanthus est particulièrement diversifié en Afrique centrale, où il a fait l'objet d'une révision récente (Sonké, 1999) qui reconnaît 17 espèces. Cheek \& Sonké (2000) ont décrit une dix-huitième espèce ultérieurement, O. okuensis Cheek \& Sonké.

En Afrique de l'Ouest (ici considérée au sens étroit, c'est-à-dire du Sénégal au Ghana), Hawthorne \& JongKIND (2006) mentionnent sept espèces d'Oxyanthus, mais leur clé n'en comprend que six. En effet, ils mettent en doute la distinction entre O. subpunctatus (Hiern) Keay et O. tenuis Stapf, deux espèces considérées comme synonymes par KeAY $(1958,1963)$ mais comme distinctes par Son KÉ (1999). Une huitième espèce, O. setosus Keay, est signalée de Côte d'Ivoire par Sonké (1999: 82); cette mention, qui repose sur une unique récolte en fruits (Breteler 6019, BR \&WAG), est passée inaperçue de HaWt horne \& JongKind (2006).

En examinant de nouveau le matériel du genre, nous avons constaté que l'échantillon Breteler 6019 appartient, avec quatre autres collections, à une espèce nouvelle bien différente d'O. setosus. Cette nouvelle espèce, O. andjigae Sonké $\&$ O. Lachenaud, est ici décrite et illustrée; nous évaluons également son statut de conservation. En outre, nous présentons une nouvelle clé du genre pour l'Afrique occidentale, et signalons pour la première fois la présence d'O. oliganthus K. Schum. en Côte d'Ivoire et au Ghana.

\section{Clé du genre Oxyanthus en Afrique de l'Ouest (du Sénégal au Ghana)}

Oxyanthus setosus n'est pas présent en Afrique de l'Ouest, mais nous l'avons inclus dans la clé car il a été signalé à tort de la région (voir la discussion ci-dessous à propos d'O. andjigae).

1. Rameaux pubescents (parfois très courtement chez O. racemosus) ....................... 2

1a. Rameaux glabres $\ldots \ldots \ldots \ldots \ldots \ldots \ldots \ldots$

2. Feuilles largement elliptiques $(>8,5 \mathrm{~cm}$ de largeur); stipules $>8 \mathrm{~mm}$ de large, obtuses ou courtement aigues; inflorescences à fleurs nombreuses (Sierra Leone - Ouganda)... . ................... Onilocularis Hiern

2a. Feuilles étroitement elliptiques ( $<8 \mathrm{~cm}$ de largeur); stipules $<5 \mathrm{~mm}$ de large, très aigues; inflorescences à fleurs peu nombreuses ...................... 3

3. Rameaux à longs poils hirsutes (1,5-2 $\mathrm{mm}$ de longueur); corolle à tube de 18-19,5 cm de longueur (Sierra Leone, Côte d'Ivoire)...... O. andjigae Sonké \& O. Lachenaud

3a. Rameaux à poils plus courts (< $1 \mathrm{~mm}$ de longueur, généralement beaucoup moins); corolle à tube de (7-)11-15,5 $\mathrm{cm}$ de longueur .................... 4

4. Feuilles coriaces, celles des rameaux florifères à base nettement asymétrique; inflorescences assez lâches, à pédicelles floraux de 3-7 mm; corolle à tube éparsement pubescent à l'extérieur; ovaire et bractées pubérulents (Guinée-Bissau - Nigeria).... O. racemosus (Schumach. \& Thonn.) Keay

4a. Feuilles minces, à base symétrique ou presque; inflorescences très condensées à pédicelles floraux nuls ou très courts $(<1,5 \mathrm{~cm})$; corolle à tube glabre extérieurement; ovaire glabre; bractées hirsutes (Nigeria - Gabon) ...... $\ldots \ldots \ldots \ldots \ldots \ldots \ldots \ldots \ldots$ O. setosus Keay

5. Inflorescence lâche et allongée; feuilles des rameaux floriferres asymétriques à la base (Mali - Ouganda)........ ............... O. formosus Hook. f. ex Planch.

5a. Inflorescence condensée; feuilles symétriques à la base . 6

6. Corolle à tube court, $3-6 \mathrm{~cm}$; inflorescences à fleurs nombreuses; nervures latérales 10-16 paires (Sénégal - Mozambique)............ O. speciosus DC. subsp. speciosus

6a. Corolle à tube $>8 \mathrm{~cm}$ de long; inflorescences à fleurs peu nombreuses, généralement moins de 10 ; nervures latérales $4-8$ paires . . . . . . . . . . . . . . . 7

7. Domaties en touffes de poils présentes aux aisselles des nervures, à la face inférieure du limbe; inflorescences à bractées bien développées. .............. 8

7a. Domaties absentes, le limbe étant entièrement glabre; inflorescences à bractées très courtes et peu visibles (Liberia - R.D. Congo) ............. O.pallidus Hiern 
8. Feuilles devenant noirâtres à sec, à nervilles invisibles ou presque; 4-6 paires de nervures latérales; fruits à pédicelles longuement accrescents, $1-2 \mathrm{~cm}$ (Côte d'Ivoire - Cameroun) ............ O. oliganthus K. Schum.

8a. Feuilles vertes ou brunes à sec, à nervilles en réseau généralement bien visible dessous; $5-8$ paires de nervures latérales; fruits à pédicelles courts, $<0,8 \mathrm{~cm}$ (Sierra Leone R.D. Congo) ...... O. subpunctatus (Hiern) Keay, s.l.

\section{Notes sur Oxyanthus oliganthus}

Cette espèce a longtemps été considérée comme endémique du Cameroun (Sonké, 1999). Récemment elle a été signalée du Gabon par Sosef et al. (2006), mais par erreur: l'unique échantillon cité, Stone et al. 3259, est en effet bien différent d'O. oliganthus par les pédicelles fructifères plus courts et le réseau de nervilles très apparent à la face inférieure du limbe; ce spécimen appartient plutôt à O. subpunctatus s.l.

En revanche, nous avons identifié récemment plusieurs récoltes d'O. oliganthus provenant de Côte d'Ivoire et du Ghana. Il s'agit des premières données pour l'Afrique occidentale et il nous a donc paru utile de documenter cette importante extension d'aire.

Les stipules d'O. oliganthus sont généralement plus petites chez les spécimens d'Afrique de l'Ouest (4-16 × 2,5-5 mm) que chez ceux du Cameroun (10-30 $\times$ 4-14 mm), mais on note un assez large recouvrement dans les dimensions. Comme, par ailleurs, tous les autres caractères correspondent, nous considérons qu'il s'agit bien du même taxon.

Oxyanthus oliganthus présente donc une aire disjointe: l'espèce se rencontre dans le sud de la Côte d'Ivoire et du Ghana (où elle est rare) et dans le sud du Cameroun entre Bipindi et la réserve du Dja (où elle est localement fréquente). Elle pousse dans les forêts de plaine: en Afrique de l'Ouest dans les forêts sempervirentes humides, et au Cameroun également en forêt semi-décidue.

Matériel étudié. - CôTe D'IvoIre. Bas-Sassandra: route de Sassandra à Monogaga, c. 455'N 6²0'W, 12.IV.1973, fl., de Koning 1462 (BR, WAG). Lagunes: Réserve Botanique de Nganda Nganda, $5 \mathrm{~km}$ au S d'Adiaké, 22.IV.1970, fl. en boutons \& fr., de Koning 338 (BR, WAG). GHANA: sine loc., s.d., Vigne 2828 (BR). Western Region: Tarkwa, Benso, IV.1951, fl., Andoh 5487 (BR).

\section{Notes sur Oxyanthus subpunctatus}

Oxyanthus subpunctatus s.l. est une espèce très polymorphe; dans l'attente d'une étude plus poussée sur sa variation, et notamment d'observations de terrain, nous admettons la synonymie d'O. tenuis proposée par KEAY $(1958,1963)$. SonKÉ (1999) avait maintenu ce dernier comme espèce distincte d'après le nombre d'inflorescences par rameau (plusieurs chez O. tenuis, une seule chez O. subpunctatus). Cependant, le type d'O. subpunctatus du Ghana (Anon. s.n., BM!) montre deux inflorescences par rameau, et paraît bien appartenir au même taxon que celui d'O. tenuis du Liberia (Whyte s.n., K!).

\section{Description de la nouvelle espèce}

La description de l'espèce est fondée sur l'étude des spécimens d'herbier conservés à $\mathrm{BR}, \mathrm{MPU}$ et WAG; toutes les dimensions indiquées concernent du matériel sec.

Oxyanthus andjigae Sonké \& O. Lachenaud, spec. nova (fig. $1 \& 2$ ).

Typus: Côte D'Ivoire. Bas-Sassandra: 10 km N de Sassandra, près de la rive gauche du fleuve Sassandra, +/- 502'N 607'W, 10.V.1975, fl, van der Burg 298 (holo-: BR!; iso-: WAG [WAG.1228171]!).

This species resembles $O$. setosus Keay and O. racemosus (Schumach. E Thonn.) Keay in the hairy twigs and narrowly elliptic leaves. However, it differs from both by the longer corolla tube (18-19.5 vs. (7-)11-15.5 cm) and the longer hairs on the twigs (1.5-2 vs. up to $1 \mathrm{~mm})$. It is further separated from $O$. racemosus by the glabrous (vs. sparsely pubescent) corolla tube and the denser inflorescences with shorter peduncles $(0-5 \mathrm{~mm}$ vs. 5-10 $\mathrm{mm}$ ); and from 0 . setosus by the distribution, the shorter calyx teeth $(0.2-1 \mathrm{~mm}$ vs. 2-2.5 mm), the puberulous ovary, the longer flowering pedicels $(3-5 \mathrm{~mm}$ vs. $0-1.5 \mathrm{~mm}$ ), the broader fruits with rounded base, and the leaves of the flowering shoots with markedly unequal base, the proximal half inserted well below the distal one (both sides inserted at same level in O. setosus).

Arbuste de 0,6-3 m. Rameaux horizontaux, à pubescence étalée blanchâtre (devenant brun clair à sec) constituée d'un mélange de longs poils hirsutes de 1,5-2 mm et de poils beaucoup plus courts, $<0,5 \mathrm{~mm}$. Stipules de 6-15 × 3-5 mm, étroitement ovées à sommet pointu, hirsutes, persistantes. Feuilles à pétiole de $0,5-1 \mathrm{~cm}$, hirsute, et limbe de 10-22 × 3,3-7,2 cm, étroitement elliptique, papyracé à légèrement coriace; base du limbe symétrique et obtuse sur les feuilles de la tige principale, très asymétrique sur celles des rameaux latéraux, avec le côté distal aigu inséré 2-6 $\mathrm{mm}$ au-dessus du côté proximal cordé ou arrondi; sommet du limbe acuminé; face supérieure glabre ou hirsute sur la nervure médiane uniquement; face inférieure entièrement couverte de longs poils hirsutes, mêlés de poils très courts sur les nervures; nervures latérales 8-11 paires, peu à moyennement ascendantes et réunies en arceaux ramifiés assez loin du bord; nervilles en réseau lâche, saillant à la face inférieure. Inflorescences pseudo-axillaires, une seule par rameau et par saison, sessiles ou à pédoncule très court $(<0,5 \mathrm{~cm})$, en grappes très contractées de 10-15 fleurs, produisant normalement un seul fruit. Bractées étroitement lancéolées, 4-8 × 0,7-1,5 mm, hirsutes. Fleurs 5-mères; pédicelles floraux de 3-5 mm, à pubérulence étalée courte. Ovaire de 1,5-3 mm, à pubérulence étalée courte. Calice à tube de 1-2 mm, glabrescent ou à pubérulence étalée courte, 


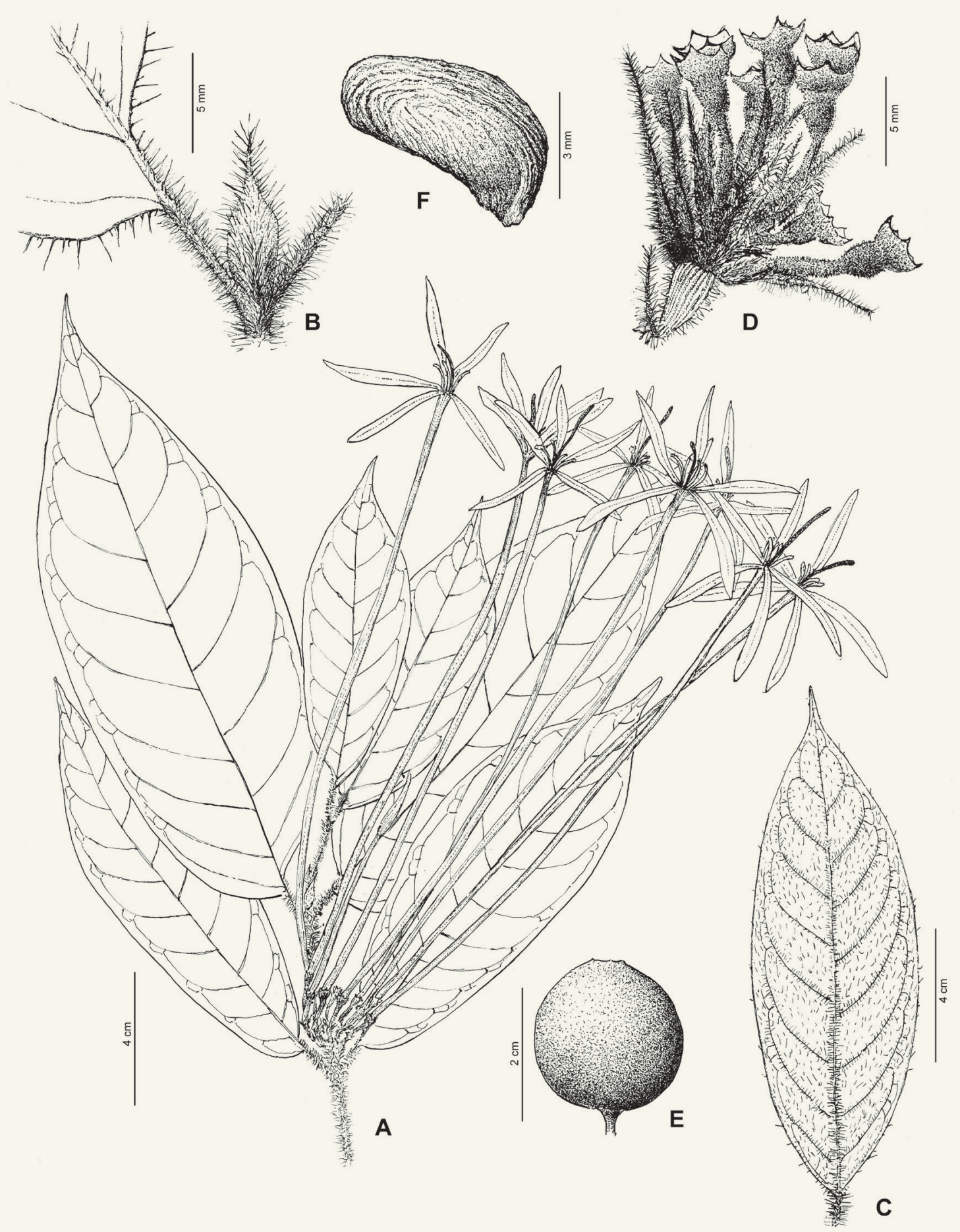

Fig. 1. - Oxyanthus andjigae Sonké \& O. Lachenaud. A. Rameau en fleurs; B. Sommet d'un rameau latéral, stipule et base d'une feuille; C. Feuille de la tige principale, face inférieure; D. Détail de l'inflorescence montrant les calices et bractées; E. Fruit; F. Graine. [A: van der Burg 298, BR; B-C, E-F: Breteler 6019, BR \& WAG; D: van der Burg 260, WAG] [Dessin: Antonio Fernandez] 


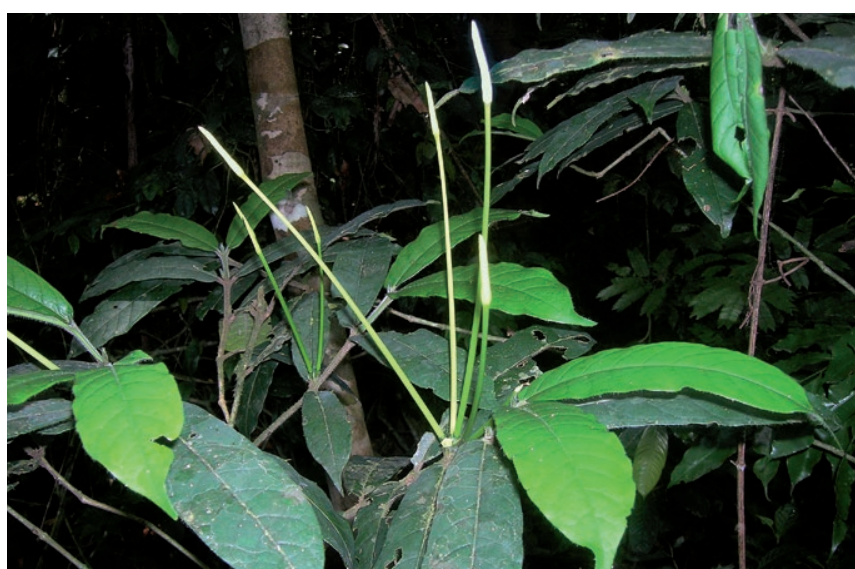

Fig. 2. - Oxyanthus andjigae Sonké \& O. Lachenaud. Plante avec fleurs en boutons. [Luke \& Rogers 15205] [Photo: Quentin Luke]

et dents triangulaires de 0,2-1 mm, glabres à courtement pubérulentes. Corolle glabre, à tube vert pâle cylindrique et très étroit de $18-19,5 \times 0,2-0,3 \mathrm{~cm}$, et lobes blanc crème étroitement lancéolés, aigus au sommet, de 2,2-3,8 × 0,2$0,4 \mathrm{~cm}$. Anthères presque entièrement exsertes, insérées à la gorge de la corolle, glabres, linéaires, 8-8,5 ×0,7 mm, à sommet apiculé sur +/- $1 \mathrm{~mm}$. Style exsert sur $+/-2,5 \mathrm{~mm}$, glabre, à stigmate allongé légèrement renflé. Fruits jaunes, ellipsoïdes à subglobuleux, arrondis aux deux bouts, 2,5-3 $\times 2-3 \mathrm{~cm}$, lisses et glabres, à pédicelle court (+/- $4 \mathrm{~mm})$ et calice caduc; nombreuses graines irrégulièrement ellipsoïdes, comprimées, +/- 6,5 × 3,5 mm, à surface ornée de stries longitudinales saillantes sur toute sa longueur.

Etymologie. - L'espèce est dédiée au Professeur Nicolas Gabriel Andjiga, Directeur de l'Ecole Normale Supérieure de l'Université de Yaoundé I, en reconnaissance de son intérêt pour la botanique, et du soutien qu'il n'a cessé d'apporter au second auteur et à toute son équipe.

Distribution et écologie. - Oxyanthus andjigae est connu d'un seul site dans le centre de la Sierra Leone, et de trois localités en Côte d'Ivoire dans la région côtière (environs de Sassandra dans le sud-ouest, et d'Assinie dans le sud-est). Cette distribution disjointe (fig. 3 ) est inhabituelle, et l'espèce serait à rechercher ailleurs en Afrique de l'Ouest, notamment au Liberia. $O$. andjigae est manifestement très rare: en effet, malgré ses fleurs très voyantes, il n'a été récolté que cinq fois. Les informations sur son habitat sont peu précises: il a été trouvé en forêt secondaire, entre 0 et $500 \mathrm{~m}$ d'altitude. Les forêts de la région de Sassandra, d'où proviennent la plupart des collections, sont de type sempervirent à Eremospatha macrocarpa G. Mann \& H. Wendl. et Diospyros mannii Hiern (Guillaumet \& Adjanohoun, 1971).

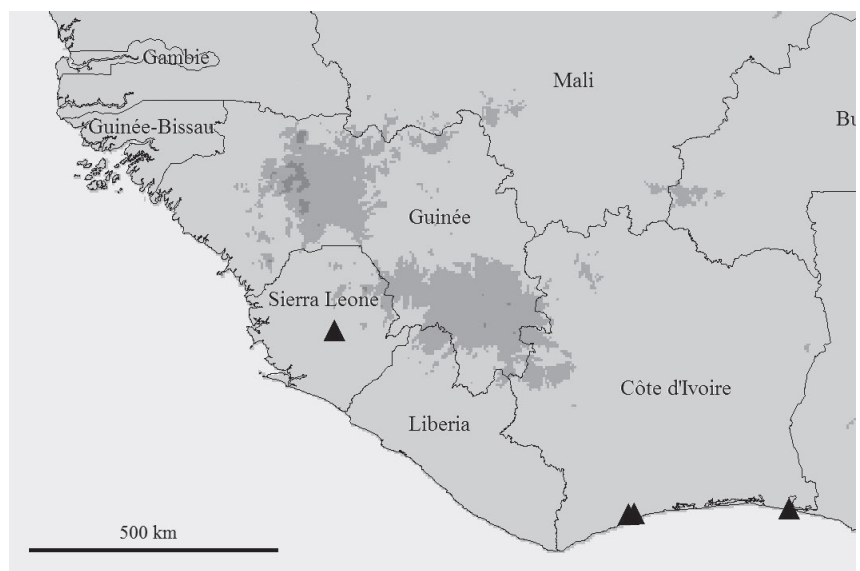

Fig. 3. - Distribution d'Oxyanthus andjigae Sonké \& O. Lachenaud.

Phénologie. - Fleurs en mai et juin; fruits en novembre.

Statut de conservation. - L'espèce est connue de quatre localités, représentant quatre sous-populations. La zone d'occurence et la zone d'occupation (calculées grâce à GeoCAT tool: BAchman et al. (2011), avec une taille de cellule fixée à $2 \times 2 \mathrm{~km}$ ) sont estimées respectivement à $67,253 \mathrm{~km}^{2}$ et $16 \mathrm{~km}^{2}$; cette dernière valeur entre dans les limites de la Catégorie «En Danger» selon le Critère B2 de la Liste Rouge de I'UICN (2012). Aucune des localités ne fait l'objet de mesures de protection. Les récoltes de l'espèce en Côte d'Ivoire sont anciennes (la dernière remonte à 1975) et il n'est pas certain que ces stations existent toujours: en effet, les régions de Sassandra et d'Assinie ont fait l'objet d'une déforestation importante pour l'établissement de plantations (et, dans le second cas, d'infrastructures touristiques) et les forêts de terre ferme qui y subsistent sont fortement fragmentées et en continuelle dégradation. La localité de Sierra Leone est quant à elle menacée par un projet minier. Un déclin de la zone d'occupation, de l'étendue et de la qualité de l'habitat, du nombre de localités, et du nombre d'individus, est donc prévisible. Oxyanthus andjigae est donc classé provisoirement comme «En Danger» [EN B2ab (ii, iii, iv, v)] selon la Liste Rouge de l'IUCN (2012). Des prospections sur le terrain devraient être conduites pour tenter de retrouver cette espèce très menacée, et si possible en effectuer des boutures pour la conservation «ex situ».

Notes. - Oxyanthus andjigae est très proche d'O. setosus Keay, avec lequel il était jusqu'à présent confondu; il ressemble aussi à 0 . racemosus (Schumach. \& Thonn.) Keay. Il se distingue toutefois assez facilement de ces deux espèces par les caractères signalés (table 1), les plus notables étant la longueur du tube de la corolle et les rameaux plus longuement pubescents. Le dimorphisme foliaire observé chez cette espèce (feuilles à base asymétrique sur les rameaux floriferres, symétriques sur la 
Table 1. - Différences entre Oxyanthus andjigae Sonké \& O. Lachenaud, O. setosus Keay, et O. racemosus (Schumach. \& Thonn.) Keay. Les caractères diagnostiques sont notés en caractères gras.

\section{O. andjigae}

\begin{tabular}{|c|c|}
\hline Pubescence des rameaux & longs poils hirsutes, $1,5-2 \mathrm{~mm}$ \\
\hline Feuilles & papyracées à légèrement coriaces \\
\hline Base du limbe & $\begin{array}{l}\text { très asymétrique sur les feuilles } \\
\text { des rameaux florifères (le côté } \\
\text { distal inséré toujours nettement } \\
\text { au-dessus de l'autre); symétrique } \\
\text { sur celles de la tige principale }\end{array}$ \\
\hline Inflorescences & $\begin{array}{l}\text { très condensées, à pédoncule nul } \\
\text { ou très court ( } 0-5 \mathrm{~mm})\end{array}$ \\
\hline Pédicelles floraux & nets, 3-5 mm \\
\hline Bractées & hirsutes \\
\hline Ovaire & densément pubérulent \\
\hline Dents du calice & $\begin{array}{l}\text { plus courtes que le tube ou l'égalan } \\
0,2-1 \mathrm{~mm}\end{array}$ \\
\hline Tube de la corolle & $\begin{array}{l}\text { très long, 18-19,5 cm, glabre } \\
\text { extérieurement }\end{array}$ \\
\hline Fruits & $\begin{array}{l}\text { arrondis aux deux bouts, } 2-3 \mathrm{~cm} \\
\text { de diamètre }\end{array}$ \\
\hline
\end{tabular}

Distribution

\section{O. setosus}

poils plus courts, 0,1-1 mm

papyracées

symétrique ou presque sur toutes les feuilles (les deux côtés insérés au même niveau)

très condensées, à pédoncule nul ou très court (0-5 mm)

nuls ou très courts, $0-1,5 \mathrm{~mm}$ (atteignant $4 \mathrm{~mm}$ en fruits)

hirsutes

glabres ou à poils très épars
O. racemosus

poils courts, 0,1-0,5 mm

coriaces

asymétrique sur les feuilles des rameaux florifères (le côté distal inséré généralement au-dessus de l'autre); symétrique sur celles de la tige principale

plus lâches, à pédoncule net (0,5-1 cm)

nets, $3-7 \mathrm{~mm}$

\section{courtement pubérulentes}

densément pubérulent

ettement plus longues que le tube, variables, $0,8-2 \mathrm{~mm}$ 2-2,5 mm

plus court, $11-12 \mathrm{~cm}$, glabre extérieurement

plus court, (7-)11-15,5 cm, éparsement pubescent extérieurement

aigus à la base, 0,9-2 cm de diamètre

arrondis ou aigus à la base, $1,2-2,4 \mathrm{~cm}$ de diamètre

du Nigeria au Gabon de la Guinée-Bissau au Nigeria tige principale) constitue une autre différence avec $O$. setosus. Un tel dimorphisme se rencontre aussi chez $O$. racemosus, et chez $O$. formosus Hook. f. ex Planch.; ce dernier est une espèce bien différente (notamment par ses feuilles et rameaux glabres, et ses inflorescences lâches).

La description de cette espèce amène à revoir la distribution d'O. setosus, qui se rencontre uniquement du Nigeria au Gabon. Le spécimen de Côte d'Ivoire, Breteler 6019, rapporté par Sonké (1999) à O. setosus, représente en fait O. andjigae.

Paratypi. - Sierra Leone. Southern Province: Bo District, Mine Site Hill, $8.4286^{\circ} \mathrm{N} 11.6623^{\circ} \mathrm{W}$, 9.VI.2011, fl., Luke \& Rogers 15205 (BR). CôTE D'IvoIRE. Bas-Sassandra: $10 \mathrm{~km} \mathrm{~N}$ de Sassandra, près de la rive gauche du fleuve Sassandra, 502'N 607’W, 10.V.1975, fl., van der Burg 260 (BR, WAG); Km 22 Sassandra - San Pedro, 501'N 6 13’W, 15.XI.1968, fr., Breteler 6019 (BR, WAG). Lagunes: Canal d'Assinie, 30.VIII.1955, st., Nozeran s.n. (MPU).

\section{Remerciements}

Les séjours de BS en Belgique en 2013 et 2014, au cours desquels la nouveauté a été découverte et décrite dans le présent article, ont été financés par l'Institut de Recherche pour le Développement (IRD) au travers du projet PEERS - ISMOBIAC. Nous tenons à remercier le Dr Pierre Couteron (Institut de Recherche pour le Développement IRD - AMAP) pour son soutien au second auteur. Nous remercions le Dr Vincent Droissart (Institut de Recherche pour le Développement IRD - AMAP) et le Dr Tariq Stévart (Missouri Botanical Garden, USA) pour leur aide dans l'évaluation du statut de conservation, ainsi que les curateurs des herbiers cités pour la mise à notre disposition de leurs collections. Nous sommes également reconnaissants à Antonio Fernandez (Jardin botanique Meise) qui a réalisé l'illustration de l'espèce. Quentin Luke nous a aimablement transmis une photographie de l'espèce et nous a fourni certains renseignements sur la localité de Sierra Leone. Nous remercions également Martin Callmander et Sylvain Razafimandimbison pour leurs commentaires pertinents sur une version antérieure du manuscrit. 


\section{Références}

Bachman, S., J. Moat, A. W. Hill, J. de la Torre \& B. Scott (2011). Supporting Red List threat assessments with GeoCAT: geospatial conservation assessment tool. In: SмiтH, V. \& L. Penev (ed.), e-Infrastructures for data publishing in biodiversity science. ZooKeys 150: 117-126.

Cheer, M. \& B. Sonké (2000). A new species of Oxyanthus (Rubiaceae-Gardeniinae) from western Cameroon. Kew Bull. 55: 889-893.

Guillaumet,J.-L. \& E. Adjanohoun (1971). La végétation de la Côte d'Ivoire. In: Avenard, J. M. et al. (ed.), Le milieu naturel de la Côte d'Ivoire: 157-266. ORSTOM, Paris.

Hallé, N. (1970). Rubiacées (2e partie). In: Aubréville, A. \& J.-F. Leroy (ed.), Fl. Gabon 17.

Hawthorne, W. \& C. C. H. Jong KInd (2006). Woody plants of Western African forests: a guide to the forest trees, shrubs and lianes from Senegal to Ghana. Royal Botanic Gardens, Kew.

IUCN (2012). IUCN Red List Categories and Criteria: Version 3.1. $2^{\text {nd }}$ ed. IUCN Species Survival Commission, Gland \& Cambridge.

KeAY, R. W. J. (1958). Randia and Gardenia in West Africa. Bull. Jard. Bot. État Bruxelles 28: 15-72.

Keay, R. W. J. (1963). Oxyanthus. In: Hepper, F. N. (ed.), Fl. W. Trop. Africa ed. 2, 2: 128-130.

Mouly, A., K. Kainulainen, C. Persson, A. P. Davis, K. M. Wong, S. G. Razafimandimbison \& B. Bremer (2014). Phylogenetic structure and clade circumscriptions in the Gardenieae complex (Rubiaceae). Taxon 63: 801-818.

Sonké, B. (1999). Oxyanthus (Rubiaceae) en Afrique centrale. Opera Bot. Belg. 8.

Sosef, M. S. M., J. J. Wieringa, C. C. H. Jongkind, G. Achoundong, Y. Azizet Issembé, D. Bedigian, R. G. van den Berg, F. J. Breteler, M. Cheek, J. Degreef, R. B. Faden, P. Goldblatt, L. J. G. van der Maesen, L. Ngok Banak, R. Niangadouma, T. Nzabi, B. Nziengui, Z. S. Rogers, T. Stévart, J. L. C. H. van Valkenburg, G. Walters \& J. J. F. E. DE WILDE (2006). Check-list des plantes vasculaires du Gabon/Checklist of Gabonese vascular plants. Scripta Bot. Belg. 35. 